\title{
Calculations of Electrets Property of Single Human Renal Stones at Regular Interval of Removing Minerals Compositions
}

\author{
R. Ramesh, J. Kishorekumar, P. Suresh and P. Sundaramoorthi* \\ Department of Physics, Thiruvalluvar Govt. Arts College,Rsipuram, Namakkal, India-637401 \\ *Corresponding Author: moorthi.sundara@gmail.com, Phone-04287-231802
}

\begin{abstract}
In the modern science and technology the electrical and thermal conductivity studies of renal stone plays an impotent role to understand the natural formation. The free electrons act as carriers to carry their energy. In the present investigations, three renal stones are collected poor hard working male who was affected with mineral deposition in the urinary tracts in a periodic collection within three years. The stones are collected from the hospital by Lithotropic treatment process. Electrical, thermal conductivities are measured at different temperatures and calculated temperature coefficients of the renal stones.
\end{abstract}

Key words: Renal stones, minerals, Process instrumentation, Bio-chemical, electron exchange and energy transfer.

\section{INTRODUCTION}

The conductivity of biomaterials, bio-minerals has been reported by the author [1]. The technical concepts such as thrombogenesis and enzymatic activity of cytochrome oxides have been dealt with either semi conductivity or electrets behavior of different bio-minerals. In the present research, both these aspects have been considered and make attempted on the same materials, namely renal stones or renal calculi. Investigation of electrets behaviors and conductivity becomes important and essential to find to inhibit the renal stones or prevention. The electrets behavioral of the renal stone material were studied through TSD, TSP [2-4]. Here the author report the D.C conductivity of renal stones as a function of temperature and applied electric field and its relations are studied. 


\section{MATERIALS AND METHODS}

Kidney stones are removed from the affected patient continuously for a period of three years by Lithotropic process in the Rasi stones diagnosing center in Rasipuram, Namakkal, India. The three stone constituents are analyzed by bio-chemical analysis process. The renal stone constituent's presences are reported in Table 1. The major constituents of the samples are calcium phosphate and its oxalate ions bonded to an organic and sulponated muscoproteins [5]. The investigation results on TSP, TSD are reproducible from stone to stone. Due to the lengthy process of research, only three samples are used in a single person (Figures 1-3).

Table 1. Chemical composition present in the renal stones.

\begin{tabular}{|c|l|}
\hline Stones & Presence of Chemical constituents \\
\hline 1 & Calcium, silica-chromium, phosphate with hydrogen oxalate group. \\
\hline 2 & Calcium oxalate, mono silica, di-hydrate with phosphate and chromium. \\
\hline 3 & Calcium oxalate with silica chromium (major) monohydrate with Phosphates. \\
\hline
\end{tabular}

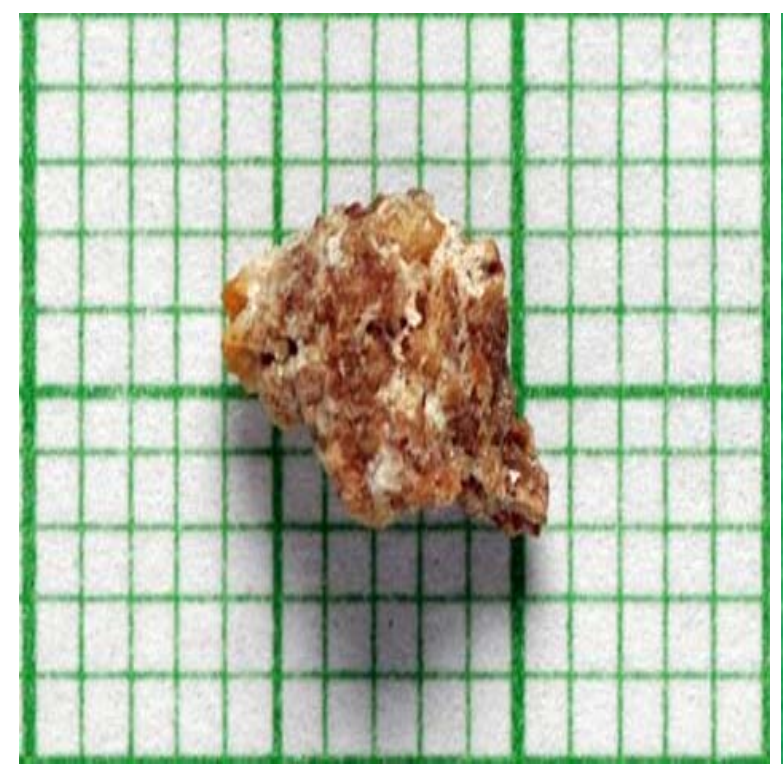

Figure 1. Stone-1.

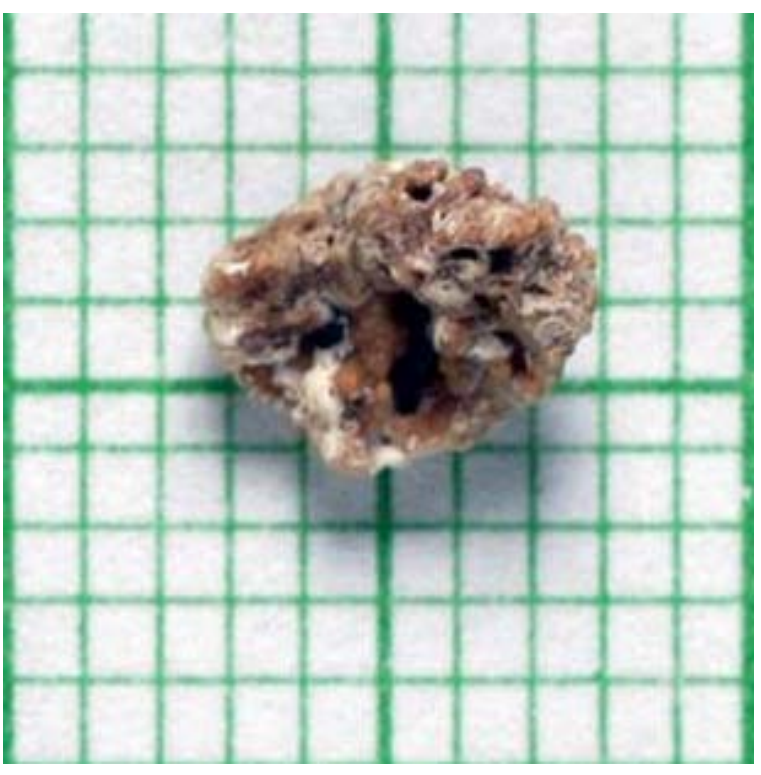

Figure 2. Stone-2. 


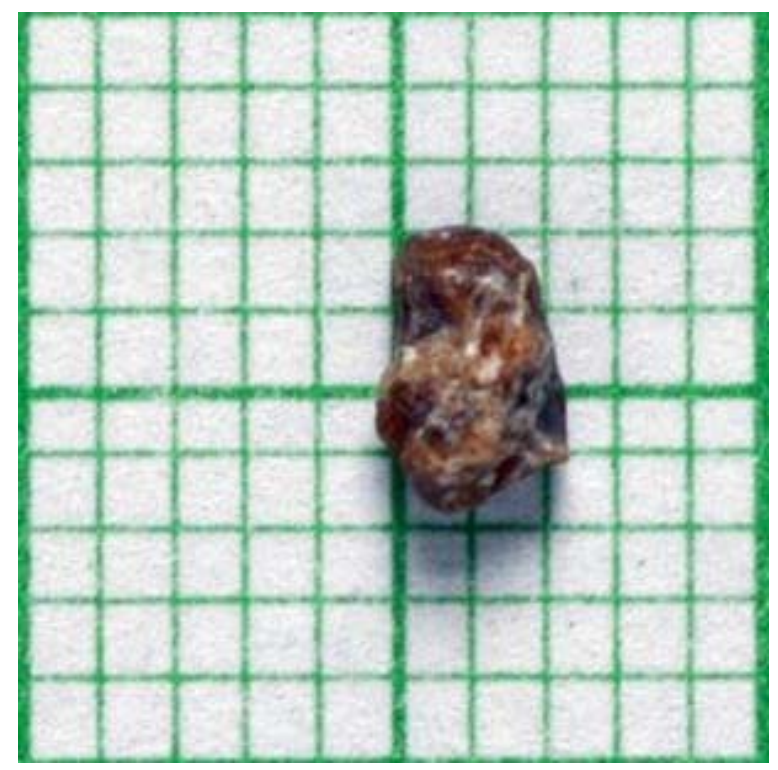

Figure 3. Stone-3.

Stone samples are initially removed in the bloodstains and other impurities from the surfaces of the sample and dried naturally. Then the renal stone sample surfaces are smoothened by using very thin grain artificial grinder. The renal stone samples are placed in the probes with in contact. The full set up are kept inside the micro oven, which is automatically heated with monitor and controller (the accuracy is $2^{\circ} \mathrm{C}$ ). The D.C conductivity is studied at the temperature (T) from 300 $\mathrm{K}$ to $370 \mathrm{~K}$ at a desired temperature. The electric field varied from 2 Volts to 16 Volts and the corresponding current flows are measured. The all the three renal stone are kept in hot Owen at a particular temperature at least 20 minutes to reach the entire heat distribution. The current flow of the renal stones mainly depends upon the presence of the carriers in a samples according to the Curie-Von-Schweidlar law. The conductivity has been reported for polymer samples [6-8] and ionic materials also [9-10].

The physical parameters of the renal stones are measured by conventional methods. The temperatures are varied from $300 \mathrm{~K}$ to $370 \mathrm{~K}$. The applied voltage $(\mathrm{V})$, corresponding current measurements $(\mathrm{I})$, resistance $(\mathrm{R})$, resistivity $(\rho)$, conductivity $(\sigma)$ and current density $(\mathrm{J})$ of each sample at a particular temperature are reported in Tables 2 to 15 . 
Table 2. Electrets properties of renal stone $A$ at $30^{\circ} \mathrm{C}, \quad A=20 \times 10^{-6} \mathrm{~m}^{2}, \mathrm{~L}=5 \times 10^{-3} \mathrm{~m}$.

\begin{tabular}{|c|c|c|c|c|c|}
\hline S. NO & $\begin{array}{l}\text { Voltage applied } \\
\text { in Volts (V). }\end{array}$ & $\begin{array}{l}\text { Current measured in } \\
\text { micro -amperes (I). }\end{array}$ & $\begin{array}{c}\text { Resistance } \\
10^{6}(\mathrm{Ohm}) .\end{array}$ & $\begin{array}{l}\text { Resistivity in } \\
\text { Ohm. m ( } \rho) .\end{array}$ & $\begin{array}{l}\text { Current } \\
\text { density (J) } \\
\mathrm{Amp} / \mathrm{m}^{2}\end{array}$ \\
\hline 1 & 2 & 4 & 0.500 & 2000 & 0.200 \\
\hline 2 & 4 & 8 & 0.500 & 2000 & 0.400 \\
\hline 3 & 6 & 12 & 0.500 & 2000 & 0.600 \\
\hline 4 & 8 & 16 & 0.500 & 2000 & 0.800 \\
\hline 5 & 10 & 18 & 0.550 & 2200 & 0.900 \\
\hline 6 & 12 & 19 & 0.632 & 2528 & 0.950 \\
\hline 7 & 14 & 20 & 0.700 & 2800 & 1.000 \\
\hline 8 & 16 & 22 & 0.727 & 2908 & 1.100 \\
\hline & \multicolumn{2}{|c|}{ Average values } & 0.576 & 2305 & 0.625 \\
\hline
\end{tabular}

Co efficient of electrical conductivity $(\sigma)=4.4338 \times 10^{-4}$ mho.m-1

Table 3. Electrets properties of renal stone $A$ at $50^{\circ} \mathrm{C}, \quad A=20 \times 10^{-6} \mathrm{~m}^{2}, \mathrm{~L}=5 \times 10^{-3} \mathrm{~m}$.

\begin{tabular}{|c|c|c|c|c|c|}
\hline S. NO & $\begin{array}{l}\text { Voltage applied } \\
\text { in Volts (V). }\end{array}$ & $\begin{array}{l}\text { Current measured } \\
\text { in micro -amperes } \\
(\mathrm{I}) .\end{array}$ & $\begin{array}{l}\text { Resistance } \\
10^{6} \\
(\mathrm{Ohm}) .\end{array}$ & $\begin{array}{l}\text { Resistivity in } \\
\text { Ohm. m ( } \rho) .\end{array}$ & $\begin{array}{l}\text { Current } \\
\text { density (J) } \\
\text { Amp/m }\end{array}$ \\
\hline 1 & 2 & 6 & 0.333 & 1332 & 0.300 \\
\hline 2 & 4 & 10 & 0.400 & 1600 & 0.500 \\
\hline 3 & 6 & 12 & 0.500 & 2000 & 0.600 \\
\hline 4 & 8 & 16 & 0.500 & 2000 & 0.800 \\
\hline 5 & 10 & 20 & 0.500 & 2000 & 1.000 \\
\hline 6 & 12 & 24 & 0.500 & 2000 & 1.200 \\
\hline 7 & 14 & 28 & 0.500 & 2000 & 1.400 \\
\hline 8 & 16 & 29 & 0.552 & 2208 & 1.450 \\
\hline & \multicolumn{2}{|l|}{ Average values } & 0.473 & 1893 & 0.906 \\
\hline
\end{tabular}

Co efficient of electrical conductivity $(\sigma)=5.2826 \times 10^{-4}$ mho.m-1 
Table 4. Electrets properties of renal stone $A$ at $75^{\circ} \mathrm{C}, \quad A=20 \times 10^{-6} \mathrm{~m}^{2}, \mathrm{~L}=5 \times 10^{-3} \mathrm{~m}$.

\begin{tabular}{|c|c|c|c|c|c|}
\hline S. NO & $\begin{array}{l}\text { Voltage applied } \\
\text { in Volts (V). }\end{array}$ & $\begin{array}{l}\text { Current measured } \\
\text { in micro -amperes } \\
(\mathrm{I}) .\end{array}$ & $\begin{array}{l}\text { Resistance } \\
10^{6} \\
(\mathrm{Ohm}) .\end{array}$ & $\begin{array}{l}\text { Resistivity in } \\
\text { Ohm. m }(\rho) .\end{array}$ & $\begin{array}{l}\text { Current } \\
\text { density }(\mathrm{J}) \\
\mathrm{Amp} / \mathrm{m}^{2}\end{array}$ \\
\hline 1 & 2 & 7 & 0.286 & 1144 & 0.350 \\
\hline 2 & 4 & 12 & 0.333 & 1332 & 0.600 \\
\hline 3 & 6 & 16 & 0.375 & 1500 & 0.800 \\
\hline 4 & 8 & 19 & 0.421 & 1684 & 0.950 \\
\hline 5 & 10 & 23 & 0.434 & 1736 & 1.150 \\
\hline 6 & 12 & 26 & 0.462 & 1848 & 1.300 \\
\hline 7 & 14 & 29 & 0.480 & 1932 & 1.450 \\
\hline 8 & 16 & 32 & 0.500 & 2000 & 1.600 \\
\hline & \multicolumn{2}{|c|}{ Average values } & 0.358 & 1430 & 0.844 \\
\hline
\end{tabular}

Co efficient of electrical conductivity $(\sigma)=6.8965 \times 10^{-4}$ mho.m-1

Table 5. Electrets properties of renal stone $A$ at $95^{\circ} \mathrm{C}, \quad A=20 \times 10^{-6} \mathrm{~m}^{2}, \mathrm{~L}=5 \times 10^{-3} \mathrm{~m}$.

\begin{tabular}{|l|l|l|l|l|l|}
\hline S. NO & $\begin{array}{l}\text { Voltage applied } \\
\text { in Volts (V). }\end{array}$ & $\begin{array}{l}\text { Current measured in } \\
\text { micro -amperes (I). }\end{array}$ & $\begin{array}{l}\text { Resistance } \\
10^{6} \\
(\mathrm{Ohm}) .\end{array}$ & $\begin{array}{l}\text { Resistivity in } \\
\text { Ohm. m ( }) .\end{array}$ & $\begin{array}{l}\text { Current } \\
\text { density (J) } \\
\text { Amp/m }\end{array}$ \\
\hline 1 & 2 & 10 & 0.2 & 800 & 0.500 \\
\hline 2 & 4 & 14 & 0.286 & 1144 & 0.700 \\
\hline 3 & 6 & 18 & 0.333 & 1332 & 0.900 \\
\hline 4 & 8 & 22 & 0.364 & 1456 & 1.100 \\
\hline 5 & 10 & 26 & 0.385 & 1540 & 1.300 \\
\hline 6 & 12 & 30 & 0.400 & 1600 & 1.500 \\
\hline 7 & 14 & 34 & 0.412 & 1648 & 1.700 \\
\hline 8 & 16 & 38 & 0.421 & 1684 & 1.900 \\
\hline & & 0.350 & 1401 & 1.013 \\
\hline
\end{tabular}

Co efficient of electrical conductivity $(\sigma)=7.1378 \times 10^{-4}$ mho.m-1 
Table 6. Electrets properties of renal stone $B$ at $33^{\circ} \mathrm{C}, \quad A=30 \times 10^{-6} \mathrm{~m}^{2}, \mathrm{~L}=5 \times 10^{-3} \mathrm{~m}$.

\begin{tabular}{|c|c|c|c|c|c|}
\hline S. NO & $\begin{array}{l}\text { Voltage applied } \\
\text { in Volts (V). }\end{array}$ & $\begin{array}{l}\text { Current measured } \\
\text { in micro -amperes } \\
\text { (I). }\end{array}$ & $\begin{array}{l}\text { Resistance } \\
10^{6} \\
(\mathrm{Ohm}) .\end{array}$ & $\begin{array}{l}\text { Resistivity in } \\
\text { Ohm. m ( } \rho) .\end{array}$ & $\begin{array}{l}\text { Current } \\
\text { density (J) } \\
\text { Amp/m }\end{array}$ \\
\hline 1 & 2 & 6 & 0.333 & 1650 & 0.200 \\
\hline 2 & 4 & 8 & 0.500 & 2500 & 0.267 \\
\hline 3 & 6 & 11 & 0.545 & 2755 & 0.367 \\
\hline 4 & 8 & 14 & 0.571 & 2855 & 0.467 \\
\hline 5 & 10 & 15 & 0.667 & 3335 & 0.500 \\
\hline 6 & 12 & 16 & 0.750 & 3750 & 0.533 \\
\hline 7 & 14 & 18 & 0.778 & 3890 & 0.600 \\
\hline 8 & 16 & 20 & 0.800 & 4000 & 0.667 \\
\hline \multicolumn{7}{|c|}{ Average values } & 0.618 & 3088 & 0.450 \\
\hline
\end{tabular}

Co efficient of electrical conductivity $(\sigma)=3.2383 \times 10^{-4}$ mho.m- 1

Table 7. Electrets properties of renal stone $B$ at $51^{\circ} \mathrm{C}, \quad A=30 \times 10^{-6} \mathrm{~m}^{2}, \mathrm{~L}=6 \times 10^{-3} \mathrm{~m}$.

\begin{tabular}{|c|c|c|c|c|c|}
\hline S. NO & $\begin{array}{l}\text { Voltage applied } \\
\text { in Volts (V). }\end{array}$ & $\begin{array}{l}\text { Current measured in } \\
\text { micro -amperes (I). }\end{array}$ & $\begin{array}{l}\text { Resistance } \\
10^{6} \\
(\mathrm{Ohm}) .\end{array}$ & $\begin{array}{l}\text { Resistivity in } \\
\text { Ohm. m ( } \rho) .\end{array}$ & $\begin{array}{l}\text { Current } \\
\text { density (J) } \\
\text { Amp/m }\end{array}$ \\
\hline 1 & 2 & 8 & 0.250 & 1250 & 0.267 \\
\hline 2 & 4 & 12 & 0.333 & 1665 & 0.400 \\
\hline 3 & 6 & 14 & 0.429 & 2145 & 0.467 \\
\hline 4 & 8 & 15 & 0.533 & 2665 & 0.500 \\
\hline 5 & 10 & 16 & 0.625 & 3125 & 0.533 \\
\hline 6 & 12 & 19 & 0.632 & 3160 & 0.633 \\
\hline 7 & 14 & 20 & 0.700 & 3500 & 0.667 \\
\hline 8 & 16 & 22 & 0.727 & 3635 & 0.733 \\
\hline \multicolumn{7}{|c|}{ Average values } & 0.528 & 2643 & 0.525 \\
\hline
\end{tabular}

Co efficient of electrical conductivity $(\sigma)=3.7835 \times 10^{-4}$ mho.m- 1 
Table 8. Electrets properties of renal stone $B$ at $74^{\circ} \mathrm{C}, \quad A=30 \times 10^{-6} \mathrm{~m}^{2}, \mathrm{~L}=6 \times 10^{-3} \mathrm{~m}$.

\begin{tabular}{|c|c|c|c|c|c|}
\hline S. NO & $\begin{array}{l}\text { Voltage applied } \\
\text { in Volts (V). }\end{array}$ & $\begin{array}{l}\text { Current measured in } \\
\text { micro -amperes (I). }\end{array}$ & $\begin{array}{c}\text { Resistance } \\
10^{6} \\
(\text { Ohm). }\end{array}$ & $\begin{array}{l}\text { Resistivity in } \\
\text { Ohm. m ( } \rho) .\end{array}$ & $\begin{array}{l}\text { Current } \\
\text { density (J) } \\
\text { Amp/m }\end{array}$ \\
\hline 1 & 2 & 12 & 0.167 & 835 & 0.400 \\
\hline 2 & 4 & 14 & 0.286 & 1430 & 0.467 \\
\hline 3 & 6 & 16 & 0.375 & 1875 & 0.533 \\
\hline 4 & 8 & 18 & 0.444 & 2200 & 0.600 \\
\hline 5 & 10 & 20 & 0.500 & 2500 & 0.667 \\
\hline 6 & 12 & 24 & 0.500 & 2500 & 0.800 \\
\hline 7 & 14 & 26 & 0.538 & 2690 & 0.867 \\
\hline 8 & 16 & 29 & 0.552 & 2760 & 0.967 \\
\hline \multicolumn{2}{|c|}{ Average values } & 0.420 & 2099 & 0.663 \\
\hline
\end{tabular}

Co efficient of electrical conductivity $(\sigma)=4.764 \times 10^{-4}$ mho.m-1

Table 9. Electrets properties of renal stone $B$ at $98^{\circ} \mathrm{C}, \quad A=30 \times 10^{-6} \mathrm{~m}^{2}, \mathrm{~L}=6 \times 10^{-3} \mathrm{~m}$.

\begin{tabular}{|c|c|c|c|c|c|}
\hline S. NO & $\begin{array}{l}\text { Voltage applied } \\
\text { in Volts (V). }\end{array}$ & $\begin{array}{l}\text { Current measured in } \\
\text { micro -amperes (I). }\end{array}$ & $\begin{array}{c}\text { Resistance } \\
10^{6} \\
(\mathrm{Ohm}) .\end{array}$ & $\begin{array}{l}\text { Resistivity in } \\
\text { Ohm. m ( } \rho) .\end{array}$ & $\begin{array}{l}\text { Current } \\
\text { density }(\mathrm{J}) \mathrm{x} \\
10^{-4} \text { Amp/m }\end{array}$ \\
\hline 1 & 2 & 14 & 0.143 & 715 & 0.467 \\
\hline 2 & 4 & 18 & 0.222 & 1110 & 0.600 \\
\hline 3 & 6 & 19 & 0.316 & 1580 & 0.633 \\
\hline 4 & 8 & 21 & 0.381 & 1905 & 0.700 \\
\hline 5 & 10 & 24 & 0.417 & 2085 & 0.800 \\
\hline 6 & 12 & 28 & 0.426 & 2130 & 0.933 \\
\hline 7 & 14 & 32 & 0.438 & 2190 & 1.070 \\
\hline 8 & 16 & 35 & 0.457 & 2285 & 1.167 \\
\hline & \multicolumn{2}{|c|}{ Average values } & 0.350 & 1750 & 0.7963 \\
\hline
\end{tabular}

Co efficient of electrical conductivity $(\sigma)=5.714 \times 10^{-4}$ mho.m-1 
Table 10. Electrets properties of renal stone $\mathrm{C}$ at $31^{\circ} \mathrm{C}, \quad A=8 \times 10^{-6} \mathrm{~m}^{2}, \mathrm{~L}=2 \times 10^{-3} \mathrm{~m}$.

\begin{tabular}{|c|c|c|c|c|c|}
\hline S. NO & $\begin{array}{l}\text { Voltage applied } \\
\text { in Volts (V). }\end{array}$ & $\begin{array}{l}\text { Current measured in } \\
\text { micro -amperes (I). }\end{array}$ & $\begin{array}{l}\text { Resistance } \\
10^{6} \\
(\mathrm{Ohm}) .\end{array}$ & $\begin{array}{l}\text { Resistivity in } \\
\text { Ohm. m ( } \rho) .\end{array}$ & $\begin{array}{l}\text { Current } \\
\text { density }(\mathrm{J}) \mathrm{x} \\
10^{-4} \text { Amp/m }\end{array}$ \\
\hline 1 & 2 & 3 & 0.667 & 2668 & 0.376 \\
\hline 2 & 4 & 5 & 0.800 & 3200 & 0.625 \\
\hline 3 & 6 & 6 & 1.000 & 4000 & 0.750 \\
\hline 4 & 8 & 8 & 1.000 & 4000 & 1.000 \\
\hline 5 & 10 & 10 & 1.000 & 4000 & 1.250 \\
\hline 6 & 12 & 12 & 1.000 & 4000 & 1.500 \\
\hline 7 & 14 & 14 & 1.000 & 4000 & 1.750 \\
\hline 8 & 16 & 16 & 1.000 & 4000 & 2.000 \\
\hline
\end{tabular}

Co efficient of electrical conductivity $(\sigma)=2.6780 \times 10^{-4}$ mho.m-1

Table 11. Electrets properties of renal stone $\mathrm{C}$ at $48^{\circ} \mathrm{C}, \quad A=8 \times 10^{-6} \mathrm{~m}^{2}, \mathrm{~L}=2 \times 10^{-3} \mathrm{~m}$.

\begin{tabular}{|c|c|c|c|c|c|}
\hline S. NO & $\begin{array}{l}\text { Voltage applied } \\
\text { in Volts (V). }\end{array}$ & $\begin{array}{l}\text { Current measured in } \\
\text { micro -amperes (I). }\end{array}$ & $\begin{array}{c}\text { Resistance } \\
10^{6} \\
(\text { Ohm). }\end{array}$ & $\begin{array}{l}\text { Resistivity in } \\
\text { Ohm. m ( } \rho) .\end{array}$ & $\begin{array}{l}\text { Current } \\
\text { density (J) x } \\
10^{-4} \text { Amp } / \mathrm{m}^{2}\end{array}$ \\
\hline 1 & 2 & 5 & 0.400 & 1600 & 0.625 \\
\hline 2 & 4 & 7 & 0.571 & 2284 & 0.875 \\
\hline 3 & 6 & 9 & 0.667 & 2668 & 1.125 \\
\hline 4 & 8 & 13 & 0.667 & 2668 & 1.625 \\
\hline 5 & 10 & 15 & 0.667 & 2668 & 1.875 \\
\hline 6 & 12 & 18 & 0.667 & 2668 & 2.250 \\
\hline 7 & 14 & 19 & 0.737 & 2948 & 2.375 \\
\hline 8 & 16 & 20 & 0.800 & 3200 & 2.500 \\
\hline \multicolumn{2}{|c|}{ Average values } & 0.647 & 3337 & 1.656 \\
\hline
\end{tabular}

Co efficient of electrical conductivity $(\sigma)=2.9967 \times 10^{-4}$ mho.m-1 
Table 12. Electrets properties of renal stone $\mathrm{C}$ at $76^{\circ} \mathrm{C}, \quad A=8 \times 10^{-6} \mathrm{~m}^{2}, \mathrm{~L}=2 \times 10^{-3} \mathrm{~m}$.

\begin{tabular}{|c|c|c|c|c|c|}
\hline S. NO & $\begin{array}{l}\text { Voltage applied } \\
\text { in Volts (V). }\end{array}$ & $\begin{array}{l}\text { Current measured in } \\
\text { micro -amperes (I). }\end{array}$ & $\begin{array}{l}\text { Resistance } \\
10^{6} \\
(\mathrm{Ohm}) .\end{array}$ & $\begin{array}{l}\text { Resistivity in } \\
\text { Ohm. m ( } \rho) .\end{array}$ & $\begin{array}{l}\text { Current density } \\
(\mathrm{J}) \times 10^{-4} \\
\text { Amp } / \mathrm{m}^{2}\end{array}$ \\
\hline 1 & 2 & 6 & 0.333 & 1332 & 0.750 \\
\hline 2 & 4 & 9 & 0.444 & 1776 & 1.125 \\
\hline 3 & 6 & 12 & 0.500 & 2000 & 1.500 \\
\hline 4 & 8 & 13 & 0.615 & 2460 & 1.625 \\
\hline 5 & 10 & 16 & 0.625 & 2500 & 2.000 \\
\hline 6 & 12 & 18 & 0.667 & 2668 & 2.250 \\
\hline 7 & 14 & 21 & 0.667 & 2668 & 2.625 \\
\hline 8 & 16 & 24 & 0.667 & 2668 & 3.000 \\
\hline
\end{tabular}

Co efficient of electrical conductivity $(\sigma)=4.4267 \times 10^{-4}$ mho.m-1

Table 13. Electrets properties of renal stone $\mathrm{C}$ at $93^{\circ} \mathrm{C}, \quad A=8 \times 10^{-6} \mathrm{~m}^{2}, \mathrm{~L}=2 \times 10^{-3} \mathrm{~m}$.

\begin{tabular}{|c|c|c|c|c|c|}
\hline S. NO & $\begin{array}{l}\text { Voltage applied } \\
\text { in Volts (V). }\end{array}$ & $\begin{array}{l}\text { Current measured in } \\
\text { micro -amperes (I). }\end{array}$ & $\begin{array}{l}\text { Resistance } \\
10^{6} \\
(\mathrm{Ohm}) .\end{array}$ & $\begin{array}{l}\text { Resistivity in } \\
\text { Ohm. m ( }) .\end{array}$ & $\begin{array}{l}\text { Current density } \\
(\mathrm{J}) \times 10^{-4} \\
\mathrm{Amp} / \mathrm{m}^{2}\end{array}$ \\
\hline 1 & 2 & 10 & 0.200 & 800 & 1.250 \\
\hline 2 & 4 & 12 & 0.333 & 1332 & 1.500 \\
\hline 3 & 6 & 16 & 0.375 & 1500 & 2.000 \\
\hline 4 & 8 & 18 & 0.444 & 1776 & 2.250 \\
\hline 5 & 10 & 19 & 0.526 & 2104 & 2.375 \\
\hline 6 & 12 & 21 & 0.571 & 2284 & 2.625 \\
\hline 7 & 14 & 24 & 0.583 & 2332 & 3.000 \\
\hline 8 & 16 & 27 & 0.593 & 2372 & 3.375 \\
\hline \multicolumn{2}{|c|}{ Average values } & 0.453 & 1813 & 2.297 \\
\hline
\end{tabular}

Co efficient of electrical conductivity $(\sigma)=5.5157 \times 10^{-4}$ mho.m-1 
Table 14. Electrical parameters of renal stone samples.

\begin{tabular}{|c|c|c|c|c|c|}
\hline S. No & Stones & $\begin{array}{l}\text { Temperature in } \\
\text { degree Celsius }\end{array}$ & $\begin{array}{r}\mathrm{R} \text { in } 10^{6} \\
\text { ohms }\end{array}$ & $\begin{array}{c}\rho \\
\text { Ohm-meter }\end{array}$ & $\begin{array}{l}\mathrm{J} \times 10^{-4} \\
\text { Ampere } / \mathrm{met}^{2}\end{array}$ \\
\hline \multirow[t]{4}{*}{1} & \multirow[t]{4}{*}{ A } & 30 & 0.576 & 2305 & 0.625 \\
\hline & & 50 & 0.473 & 1893 & 0.906 \\
\hline & & 75 & 0.358 & 1430 & 0.844 \\
\hline & & 95 & 0.350 & 1401 & 1.013 \\
\hline \multirow[t]{4}{*}{2} & \multirow[t]{4}{*}{$\mathrm{B}$} & 32 & 0.618 & 3088 & 0.450 \\
\hline & & 51 & 0.528 & 2643 & 0.525 \\
\hline & & 74 & 0.420 & 2099 & 0.663 \\
\hline & & 98 & 0.350 & 1750 & 0.7963 \\
\hline \multirow[t]{4}{*}{3} & \multirow[t]{4}{*}{$\mathrm{C}$} & 31 & 0.933 & 3734 & 1.156 \\
\hline & & 48 & 0.647 & 3337 & 1.656 \\
\hline & & 76 & 0.565 & 2259 & 1.859 \\
\hline & & 97 & 0.453 & 1813 & 2.297 \\
\hline
\end{tabular}

Table 15. Thermal conductivity of renal stones at different temperature.

\begin{tabular}{|c|c|c|c|c|}
\hline S. No & $\begin{array}{l}\text { Name of the } \\
\text { (Stone) } \\
\text { samples }\end{array}$ & $\begin{array}{l}\text { Conductivity } \\
(\sigma) \times 10^{-4} \\
\text { mho.m-1 }\end{array}$ & $\begin{array}{c}\text { Temperature } \\
\text { in degree Celsius }\end{array}$ & $\begin{array}{c}\text { Thermal } \\
\text { conductivity } \\
\mathrm{K}=\underset{\mathrm{W} / \mathrm{mc}}{\sigma \mathrm{L}_{\mathrm{z}} \mathrm{T}} \mathrm{X} 10^{-10}\end{array}$ \\
\hline \multirow[t]{4}{*}{1} & \multirow[t]{4}{*}{ A } & 4.4338 & 30 & 1.4898 \\
\hline & & 5.2826 & 50 & 2.9583 \\
\hline & & 6.8965 & 75 & 5.7931 \\
\hline & & 7.1378 & 95 & 7.5941 \\
\hline \multirow[t]{4}{*}{2} & \multirow[t]{4}{*}{ B } & 3.2383 & 32 & 1.1606 \\
\hline & & 3.7835 & 51 & 2.1611 \\
\hline & & 4.7640 & 74 & 3.9484 \\
\hline & & 5.7140 & 98 & 6.2717 \\
\hline \multirow[t]{4}{*}{3} & \multirow[t]{4}{*}{$\mathrm{C}$} & 2.6780 & 31 & 0.9298 \\
\hline & & 2.9967 & 48 & 1.6110 \\
\hline & & 4.4267 & 76 & 4.1044 \\
\hline & & 5.5157 & 97 & 6.5272 \\
\hline
\end{tabular}


One can unable to measure the hall coefficient of the renal stones. The stones containing collagens like micro-protein and apatite also have some organic matrix like protein at $5 \%$ of the total weight [13]. The protein matrix is clearly visible under a scanning microscope. The D.C electrical conductivity of the renal stones are compared and calculated with the standard ionic conductors [11] and semiconductors [12].

The ionic conduction for the renal stone is:

$$
\sigma=\sigma_{1} \exp ^{(\mathrm{A} / \mathrm{KT})}+\sigma_{2} \exp ^{(-\mathrm{B} / \mathrm{KT})}
$$

Where $\sigma_{1}, \sigma_{2}$ are the zero field conductivity, A and B are constants. The two exponential terms are nature of normal conductors.

In semiconductors the conductivity,

$$
\sigma=\sigma_{0} \exp ^{(-\mathrm{Eg} / 2 \mathrm{KT})}
$$

Here $\sigma_{0}$ is the zero field conductivity and Eg is the activation energy of the conductors at a particular temperature (T). Because of practical inconvenience of electron microscope (SEM), a fine powder of kidney stone was observed under a high resolution optical microscope and shows fibrils of protein. Thus renal stones can be regarded as a mixture of semi conducting materials like $\mathrm{N}$ type and $\mathrm{P}$ type, but totally it behaves like $\mathrm{N}$ type material or conductors. Hence, conductivity of a kidney stone shows it may be interpreted in terms of a partially compensated semiconductor. Available mechanism for conduction of renal stones may be sought with the help of various scattering mechanism of conductors and semiconductors [14]. The conductivity of samples depends upon the scattering by lattice vibrations. In conductor the curve between conductivity and temperature should be straight line, but in semiconductor, it is not in usual [15].

The TSP, TSD data of kidney stones also give added information about its conduction mechanism $[2,3]$. For the TSP, TSD conductivity of a sample, the changing current is composed of three components, which are conduction, polarization and depolarization. When temperature increases, the conductivity of a samples is increased, the polarization and depolarization peaks merge in the conduction current or only a part of it is observable [16]. The voltage dependence of conductivity decreases with rise of temperature $(\mathrm{T})$. The current density decreases, which shows the conduction is in non-ohmic. This change suggests a warm electron effect [17]. At higher temperature, the current density is directly proportional to voltage and gives the ohmic behaviors. This is clear that for a sample at higher temperature, the thermal energy difference between the charge carriers and lattices are relatively low or due to asymmetric effect [18] formed in inside the crystals of calcium oxalates and calcium phosphates. 


\section{CONCLUSION}

For all the three renal stone samples, thermal conductivity and electrical conductivity are calculated. At higher temperature, the thermal conductivity of all the samples is increased and the electrical conductivity changed. The entire samples are high conductivity at low temperature, but when the temperature increases, the conductivity decreases.

\section{REFERENCES}

[1] C.Simionescu, et al., Topics in biochemistry and Biogenetics, John Willy and sons, New York, 27, 151-204 (1976).

[2] Ya. Yagik, etal., Solid State Phys Sym. BARC, Bombay, India, 23-25, 27c., 205 (1984).

[3] Ya.Yagik, etal., Bioelectrets of renal calculi (kidney stone) in proc. $5^{\text {th }}$ Int. Sym. Electrets Heidelberg, 819-824 (1985)

[4] Ya.Yagik, etal, 8(b), 503-505 (1987).

[5] C.W.Vermuclen, etal., J.urology, 95,681 (1964).

[6] J. Lowell, J. Phys. D, 15,109 (1982).

[7] I.M.Talwar, etal., Ind.J.Pure.Appl.Phys., 7, 681 (1982).

[8] B. Sharma, Polymer, 73, 17 (1987).

[9] J.Consh, etal., J.Phys.Chem.solids, 33, 1799 (1972).

[10] C.Ramasastry, etal., Proc.R.Soc. A 325, 34715.(1971)

[11] A.Vanderzerd, Solid state physical Electronics printer's hall of India, New Delhi, 492 (1971).

[12] R.Pething, Dielectrics \& electronic properties of biological materials. John Willy and sons, New York., 303 (1979).

[13] R.K.Jethi, etal., Ind. J medical research, 78, 422-25 (1983).

[14] P.S.Kareev, Semiconductor physics Mir publishers, Moscow, 428-436 (1974).

[15] P.S.Greeve, Physics and technology of semiconductor devices. John Willy and sons, Newyork, 109 (1967).

[16] S.W.S.Mckeever, et al., J.phy. D, 8, 1520 (1975).

[17] J.S.Black more, Solid state physics $2^{\text {nd }}$ edi. W.B. Sawnders Company Tokyo, Japan, 357358 (1974).

[18] S.Glasstone, An introduction to electro chemistry D.Van No strand, 878-114 (1965). 